\title{
Erratum to: The Alteration of Zinc Transporter Gene Expression Is Associated with Inflammatory Markers in Obese Women
}

\author{
Hwayoung Noh • Hee Young Paik • Jihye Kim • \\ Jayong Chung
}

Published online: 6 June 2014

(C) Springer Science+Business Media New York 2014

Erratum to: Biol Trace Elem Res (2014) 158:1-8

DOI 10.1007/s12011-014-9902-1

The Editors regret that there are errors in Table 3 in the article by Noh et al: "The Alteration of Zinc Transporter Gene Expression is Associated with Inflammatory Markers in Obese Women," Biol Trace Elem Res (2014) 158:1-8, DOI 10.1007/s12011-014-9902-1. Three asterisks indicating $p<0$. 001 belong only in rows 1 and 2 (BMI and body fat), columns 1-3 (ZnT4, ZnT5, ZnT7) and columns 4, 5 (Zip1, Zip4). The correct Table 3 is presented here.

The online version of the original article can be found at http://dx.doi.org/ 10.1007/s12011-014-9902-1.

H. Noh $\cdot$ H. Y. Paik

Department of Food and Nutrition, College of Human Ecology,

Seoul National University, Seoul 151-742, South Korea

J. $\operatorname{Kim}(\bowtie)$

Department of Medical Nutrition, Graduate School of East-West Medical Science, Kyung Hee University, 1732 Deogyeong-daero, Giheung-gu, Yongin-si, Gyeonggi-do 446-701, South Korea e-mail: kjhye@khu.ac.kr

J. Kim

Research Institute of Medical Nutrition, Kyung Hee University,

Seoul 130-701, South Korea

J. Chung $(\square)$

Department of Food and Nutrition, College of Human Ecology,

Kyung Hee University, 26 Kyunghee-daero Dongdaemun-gu,

Seoul 130-701, South Korea

e-mail: jchung@khu.ac.kr 
Table 3 Spearman's correlation coefficients among the mRNA levels of leukocyte zinc transporters, anthropometric measurements, zinc status, inflammatory markers, and biochemical parameters in subjects $(n=55)^{\mathrm{a}}$

\begin{tabular}{|c|c|c|c|c|c|c|c|}
\hline & ZnT4 & ZnT5 & $\mathrm{ZnT7}$ & ZnT9 & Zip1 & Zip4 & Zip6 \\
\hline \multicolumn{8}{|l|}{ Anthropometric measurements } \\
\hline BMI $\left(\mathrm{kg} / \mathrm{m}^{2}\right)$ & $-0.70^{* * *}$ & $-0.59^{* * *}$ & $0.57^{* * *}$ & $-0.31^{*}$ & $-0.65^{* * *}$ & $-0.50^{* * *}$ & $-0.27^{*}$ \\
\hline Body fat (\%) & $-0.67^{* * *}$ & $-0.60^{* * *}$ & $0.58^{* * *}$ & $-0.29^{*}$ & $-0.61^{* * *}$ & $-0.53^{* * *}$ & -0.20 \\
\hline \multicolumn{8}{|l|}{ Zinc status $^{\mathrm{a}}$} \\
\hline Zinc intake (mg/d) & 0.02 & -0.02 & 0.11 & 0.03 & 0.18 & 0.08 & 0.16 \\
\hline Serum zinc $(\mu \mathrm{mol} / \mathrm{L})$ & -0.04 & -0.11 & 0.00 & -0.04 & 0.12 & 0.06 & 0.00 \\
\hline Urine zinc (ug/mg creatinine) & 0.24 & 0.26 & 0.21 & $0.32^{*}$ & 0.07 & 0.18 & $0.31^{*}$ \\
\hline $\mathrm{SOD}(\mathrm{U} / \mathrm{mL})$ & -0.02 & 0.09 & -0.16 & 0.00 & 0.15 & 0.10 & -0.08 \\
\hline \multicolumn{8}{|l|}{ Inflammatory markers $^{\mathrm{a}}$} \\
\hline hs-CRP (nmol/L) & $-0.30^{*}$ & -0.23 & -0.08 & -0.10 & $-0.32^{*}$ & 0.04 & $-0.29^{*}$ \\
\hline $\mathrm{TNF} \alpha(\mathrm{pg} / \mathrm{mL})$ & $-0.30^{*}$ & $-0.28^{*}$ & -0.11 & -0.05 & -0.25 & -0.01 & -0.11 \\
\hline IL-6 (pg/mL) & 0.19 & 0.10 & -0.03 & 0.16 & 0.20 & 0.22 & 0.07 \\
\hline \multicolumn{8}{|l|}{ Biochemical parameters $^{\mathrm{a}}$} \\
\hline Triacylglycerol(mmol/L) & -0.03 & -0.13 & 0.27 & -0.11 & -0.17 & 0.03 & 0.05 \\
\hline Total cholesterol $(\mathrm{mmol} / \mathrm{L})$ & 0.10 & 0.01 & 0.22 & -0.19 & -0.19 & 0.16 & 0.01 \\
\hline Insulin (pmol/L) & 0.01 & 0.07 & 0.00 & 0.00 & -0.19 & 0.18 & -0.01 \\
\hline Adiponectin $(\mu \mathrm{g} / \mathrm{mL})$ & -0.05 & -0.01 & -0.05 & -0.02 & 0.01 & 0.03 & -0.02 \\
\hline Leptin $(\mu \mathrm{g} / \mathrm{L})$ & -0.20 & -0.18 & 0.21 & 0.05 & -0.03 & -0.01 & 0.12 \\
\hline
\end{tabular}

$S O D$ superoxide dismutase, $h s-C R P$ high sensitivity C-reactive protein, $T N F \alpha$ tumor necrosis factor-alpha, IL-6 interleukin-6

${ }^{a}$ Adjusted for BMI by Spearman's partial correlation analysis $* P<0.05$; *** $P<0.001$ 